independent forms. J. Immunol. 161:1710-1717.

11. Yanaba, K., et al. 2008. A regulatory B cell subset with a unique $\mathrm{CD} 1 \mathrm{dhiCD} 5+$ phenotype controls $\mathrm{T}$ cell-dependent inflammatory responses. Immunity. 28:639-650

12. Wang, B., et al. 2000. CD4+ Th1 and CD8+ type 1 cytotoxic $\mathrm{T}$ cells both play a crucial role in the full development of contact hypersensitivity. J. Immunol. 165:6783-6790.

13. Fillatreau, S., Sweenie, C.H., McGeachy, M.J., Gray, D., and Anderton, S.M. 2002. B cells regulate autoimmunity by provision of IL-10. Nat. Immunol. 3:944-950

14. Mizoguchi, A., Mizoguchi, E., Takedatsu, H., Blumberg, R.S., and Bhan, A.K. 2002. Chronic intestinal inflammatory condition generates IL-10-producing regulatory $\mathrm{B}$ cell subset characterized by CD1d upregulation. Immunity. 16:219-230.
15. Mauri, C., Gray, D., Mushtaq, N., and Londei, M. 2003. Prevention of arthritis by interleukin 10-producing B cells. J. Exp. Med. 197:489-501.

16. Duddy, M.E., Alter, A., and Bar-Or, A. 2004. Distinct profiles of human B cell effector cytokines: a role in immune regulation? J. Immunol. 172:3422-3427.

17. Duddy, M., et al. 2007. Distinct effector cytokine profiles of memory and naive human B cell subsets and implication in multiple sclerosis. J. Immunol. 178:6092-6099.

18. Moulin, V., et al. 2000. B lymphocytes regulate dendritic cell (DC) function in vivo: increased interleukin 12 production by DCs from B cell-deficient mice results in T helper cell type 1 deviation. J. Exp. Med. 192:475-482.

19. Lampropoulou, V., et al. 2008. TLR-activated B cells suppress $\mathrm{T}$ cell-mediated autoimmunity. J. Immunol. 180:4763-4773.
20. Mann, M.K., Maresz, K., Shriver, L.P., Tan, Y., and Dittel, B.N. 2007. B cell regulation of CD4+CD25+ $T$ regulatory cells and IL-10 via B7 is essential for recovery from experimental autoimmune encephalomyelitis. J. Immunol. 178:3447-3456.

21. Abdul-Majid, K.B., et al. 2002. Fc receptors are critical for autoimmune inflammatory damage to the central nervous system in experimental autoimmune encephalomyelitis. Scand. J. Immunol. 55:70-81.

22. Lyons, J.A., Ramsbottom, M.J., and Cross, A.H 2002. Critical role of antigen-specific antibody in experimental autoimmune encephalomyelitis induced by recombinant myelin oligodendrocyte glycoprotein. Eur. J. Immunol. 32:1905-1913.

23. Goetz, M., Atreya, R., Ghalibafian, M., Galle, P.R., and Neurath, M.F. 2007. Exacerbation of ulcerative colitis after rituximab salvage therapy. Inflamm. Bowel Dis. 13:1365-1368.

\title{
Hedgehog signaling in biliary fibrosis
}

\author{
Linda E. Greenbaum
}

Division of Gastroenterology, Department of Medicine, University of Pennsylvania School of Medicine, Philadelphia, Pennsylvania, USA.

\begin{abstract}
Congenital and acquired diseases of the biliary tree, or cholangiopathies, represent a significant source of morbidity and mortality in both children and adults. In late stages of the disease, cholangiocytes can no longer proliferate, resulting in loss of bile ducts, increased fibrosis, and ultimately cirrhosis and liver failure. Epithelial-mesenchymal transition has been proposed as a potential mechanism underlying both cholangiocyte proliferation and fibrogenesis in biliary diseases. In this issue of the JCI, using a myofibroblast-cholangiocyte coculture system and genetically modified mice, Omenetti and colleagues present evidence supporting the importance of paracrine hedgehog signaling between the two cell types and increased expression of mesenchymal markers in cholangiocytes (see the related article beginning on page 3331). These findings set the stage for future studies to further investigate the contribution of hedgehog signaling in both cholangiocyte repair and fibrogenesis in biliary diseases.
\end{abstract}

Cholangiopathies represent a spectrum of both congenital and acquired disorders characterized by chronic bile duct injury (reviewed in refs. 1, 2). Although cholangiocytes initially have the ability to proliferate in response to cellular injury, latestage cholangiopathies are associated with loss of bile ducts and progressive fibrosis, resulting in cirrhosis and liver failure.

Ductular reactions, composed of small and apparently proliferating cholangiocytes in the periportal region, are detected in many forms of chronic liver injury, including those that primarily effect cholangiocytes. These cholangiocytes have

Nonstandard abbreviations used: EMT, epithelialmesenchymal transition; PBC, primary biliary cirrhosis.

Conflict of interest: The author has declared that no conflict of interest exists.

Citation for this article: J. Clin. Invest. 118:3263-3265 (2008). doi:10.1172/JCI37189. been termed reactive cholangiocytes, and they acquire phenotypic behaviors not observed in the noninjured state, including secretion of inflammatory cytokines, chemoattractant proteins, and inhibitors of apoptosis. Reactive cholangiocytes are believed to arise from a progenitor cell compartment located along the terminal bile ductules and in the canal of Hering (2). However, the origin of these reactive cholangiocytes has not yet been established definitively. Evidence from experimental animal models has shown that crosstalk among reactive cholangiocytes, myofibroblasts, endothelial cells, and inflammatory cells contributes to cholangiocyte proliferation as well as the fibrotic response $(1,2)$. The identity and relative contribution of these paracrine mediators and the mechanisms that directly regulate cholangiocyte proliferation and fibrogenesis remain incompletely understood.
Potential role of epithelialmesenchymal transition in cholestatic liver injury

In addition to secreting numerous regulatory molecules in response to cholangiocyte injury, reactive cholangiocytes lose some of their epithelial phenotypic characteristics and acquire a more mesenchymal phenotype characterized by the loss of E-cadherin and increased expression of some mesenchymal markers. During epithelial-mesenchymal transition (EMT), epithelial cells lose polarity and cell-cell communication and acquire migratory and invasive phenotypic cellular behaviors. This process is important for tissue remodeling during normal development and more recently has been recognized as a component of repair processes in fibrotic diseases and malignancy $(3,4)$. EMT has been implicated in fibrotic diseases of the biliary tract as a potential mechanism for disappearance of bile ductules and concomitant increase in fibrosis (5-7). How EMT might be regulated in chronic biliary diseases is not known.

Previously, Omenetti and colleagues reported that the hedgehog signaling pathway is activated in both cholangiocytes and fibroblastic cells in experimental models of biliary injury and in livers of patients with primary biliary cirrhosis $(\mathrm{PBC})(8,9)$. Because the hedgehog signaling pathway is known to be a positive effector of EMT in other tissues, the authors hypothesized that hedgehog signaling pathways could also be involved in EMT in response to cholestatic liver injury and fibrosis $(10,11)$. 


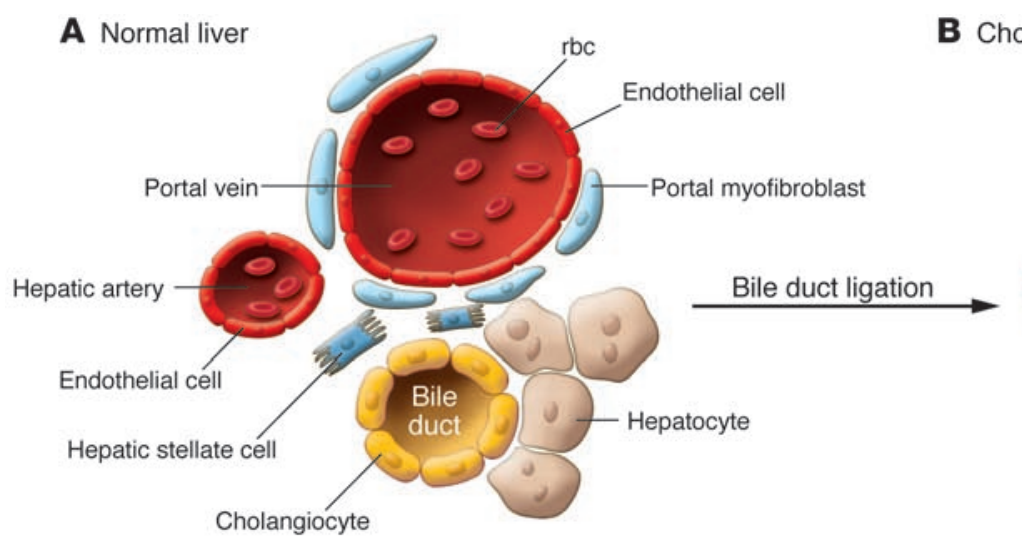

Model of paracrine signaling in a ductular reaction in the cholestatic liver. (A) Schematic of the portal triad of the portal vein, hepatic artery, and bile duct in the normal liver. Hepatic stellate cells, the site of vitamin A storage, are located in the Disse space near hepatocytes. Portal myofibroblasts are also in close proximity to the bile ducts. (B) After bile duct ligation and cholestatic liver injury, the liver responds with a ductular reaction and the expansion of the biliary tree. In their study in this issue of the $\mathrm{JCl}$, Omenetti and colleagues (12) propose that hepatic stellate cells produce and release sonic hedgehog $(\mathrm{Hh})$ in response to the injury, which acts on a subpopulation of bile duct epithelial cells known as cholangiocytes to activate several markers of mesenchymal cells. The authors suggest that cholangiocytes then undergo EMT and migrate into the parenchyma. The latter hypothesis remains to be proven in vivo. Also, additional signals from the portal myofibroblasts likely contribute to the activation of bile duct epithelial cells.

The study by Omenetti and colleagues in the current issue of the JCI (12) tests this hypothesis, demonstrating that the mesenchymal marker S100A4 is expressed in a subset of ductular and fibroblastic cells in livers of patients with PBC, confirming previous findings $(5,6)$. Using a rat model of reversible chronic bile duct obstruction, they further show that expression of this mesenchymal marker is induced following bile duct obstruction. They confirm that this activation of a mesenchymal marker occurs within cholangiocytes by demonstrating both upregulation of mesenchymal markers and decreased expression of epithelial markers in cholangiocytes isolated from the livers of bile duct-ligated rats. Using the same approach, they demonstrate that cholangiocytes isolated from bile duct-ligated livers express hedgehog proteins and these hedgehog proteins are coexpressed with mesenchymal markers in ductular cells from the livers of patients with PBC.

These findings define a novel association between the expression of hedgehog proteins and mesenchymal markers in cholangiocytes after bile duct injury (12). To establish a functional relationship between hedgehog proteins and EMT, the authors used an in vitro system in which a cholangiocyte cell line is cocultured with a hepatic stellate cell line to test whether paracrine hedgehog signaling not only affects expression of mesenchymal markers but also increases the migratory behavior of cholangiocytes. They demonstrate that soluble factors expressed by hepatic stellate cells induce expression of mesenchymal markers in cholangiocytes. More importantly, using carefully performed cell migration assays, the authors show that soluble factors from hepatic stellate cells increase cholangiocyte motility and migration, two hallmarks of the mesenchymal phenotype. When conditioned medium from hepatic stellate cells was treated with hedgehog-neutralizing antibodies, the expression of mesenchymal markers and cholangiocyte migratory behavior were significantly reduced. This experiment provides what is believed to be the first functional evidence that hepatic stellate cells can influence cholangiocyte migratory behavior through a mechanism that is dependent upon hedgehog-mediated paracrine signaling (Figure 1).

To investigate whether alterations in hedgehog signaling are associated with increased EMT in vivo, Omenetti and colleagues performed bile duct ligation on mice haploinsufficient for the hedgehog inhibitor patched (Ptc) (12); these mice exhibit increased hedgehog signaling activity. As predicted, the livers of the mice showed enhanced fibrogenesis in response to bile duct ligation and elevated expression of the hedgehog transcription factor Gli2 and several mesenchymal markers. In the future, it will be important to establish that the change in cellular behavior of the cholangiocyte cell line, especially with respect to cell migration, seen in vitro can also be linked to hedgehog signaling in vivo.

\section{Future directions}

The current study provides compelling evidence of hepatic stellate cell secretion of hedgehog proteins that induce expression of mesenchymal marker genes and increase cholangiocyte migration in a coculture system (12). While Omenetti and colleagues demonstrate that increased hedgehog signaling in vivo is associated with increased fibrogenesis, the mechanism by which this occurs remains to be addressed. It is likely that hedgehog-mediated activation of cholangiocytes during bile duct injury results in the secretion of soluble factors that in turn enhance activation of hepatic stellate and other myofibroblastic cells, resulting in increased fibrogenesis. To explore this hypothesis, it might be possible to utilize the hepatic stellate cell/cholangiocyte system to identify factors secreted by cholangiocytes that result in hepatic stellate cell and portal fibroblast activation.

An important concept raised by the current study (12) stems from the observation 
that some of the cholangiocytes within the ductular reactions costained for Gli2 and either the mesenchymal marker S100A4 or vimentin. It is plausible that EMT occurs in a subset of reactive cholangiocytes within the ductular reaction. As proposed by the authors, ductular cells that respond to hedgehog signaling may represent a hepatic progenitor cell population capable of differentiating into fibrogenic cell types in response to liver injury. Support for this comes from cell culture studies indicating that cholangiocytes are capable of this type of transformation in vitro (6). In addition, Yovchev and colleagues have isolated a subset of hepatic progenitor cells that express both epithelial and mesenchymal markers, including several markers of EMT that are capable of repopulating injured rat liver (13). It would be useful to investigate whether the progenitor cells isolated in their study also respond to hedgehog signaling in a coculture system. Such evidence would provide support for the notion that hedgehog-responsive ductular cells represent a hepatic progenitor cell population. However, definitive proof that cholangiocytes are able to transdifferentiate into myofibroblasts will require in vivo lineage tracing with cholangiocyte-specific marker genes.

We can conclude from this study (12) that hedgehog signaling is likely to medi- ate both beneficial and deleterious effects of liver injury, depending upon the balance between its action as a survival factor for cholangiocytes and as a profibrogenic agent. Further characterization of the mechanisms of hepatic repair regulated by the hedgehog pathway and potential synergistic interaction with other signaling pathways involved in both cholangiocyte proliferation and fibrogenesis will be necessary prior to attempting to enhance or inhibit hedgehog signaling in chronic fibrotic liver diseases.

\section{Acknowledgments}

I thank Rebecca Wells, Klaus H. Kaestner, and Joshua R. Friedman for helpful suggestions on the manuscript.

Address correspondence to: Linda E. Greenbaum, Division of Gastroenterology, Department of Medicine, University of Pennsylvania, 600 Clinical Research Building, 415 Curie Boulevard, Philadelphia, Pennsylvania 19104, USA. Phone: (215) 573-1868; Fax: (215) 573-2024; E-mail: greenbal@mail.med.upenn.edu.

1. Xia, X., et al. 2007. Cholangiocyte injury and ductopenic syndromes. Semin. Liver Dis. 27:401-412.

2. Lazaridis, K.N., Strazzabosco, M., and Larusso, N.F. 2004. The cholangiopathies: disorders of biliary epithelia. Gastroenterology. 127:1565-1577.
3. Lee, J.M., Dedhar, S., Kalluri, R., and Thompson, E.W. 2006. The epithelial-mesenchymal transition: new insights in signaling, development, and disease. J. Cell Biol. 172:973-981.

4. Baum, B., Settleman, J., and Quinlan, M.P. 2008. Transitions between epithelial and mesenchymal states in development and disease. Semin. Cell Dev. Biol. 19:294-308.

5. Robertson, H., Kirby, J.A., Yip, W.W., Jones, D.E., and Burt, A.D. 2007. Biliary epithelial-mesenchymal transition in posttransplantation recurrence of primary biliary cirrhosis. Hepatology. 45:977-981.

6. Rygiel, K.A., et al. 2008. Epithelial-mesenchymal transition contributes to portal tract fibrogenesis during human chronic liver disease. Lab. Invest. 88:112-123.

7. Diaz, R., et al. 2008. Evidence for the epithelial to mesenchymal transition in biliary atresia fibrosis. Hum. Pathol. 39:102-115.

8. Omenetti, A., et al. 2007. Hedgehog-mediated mesenchymal-epithelial interactions modulate hepatic response to bile duct ligation. Lab. Invest. 87:499-514.

9. Omenetti, A., et al. 2008. The hedgehog pathway regulates remodeling responses to biliary obstruction in rats. Gut. 57:1275-1282.

10. Huber, M.A., Kraut, N., and Beug, H. 2005. Molecular requirements for epithelial-mesenchymal transition during tumor progression. Curr. Opin. Cell Biol. 17:548-558.

11. Li, X., Deng, W., Lobo-Ruppert, S.M., and Ruppert, J.M. 2007. Gli1 acts through Snail and E-cadherin to promote nuclear signaling by beta-catenin. Oncogene. 26:4489-4498.

12. Omenetti, A., et al. 2008. Hedgehog signaling regulates epithelial-mesenchymal transition during biliary fibrosis in rodents and humans. J. Clin. Invest. 118:3331-3342.

13. Yovchev, M.I., et al. 2008. Identification of adult hepatic progenitor cells capable of repopulating injured rat liver. Hepatology. 47:636-647.

\title{
Prenatal maternal diet affects asthma risk in offspring
}

\author{
Rachel L. Miller \\ Division of Pulmonary, Allergy, and Critical Care Medicine, Columbia University College of Physicians and Surgeons, New York, New York, USA.
}

\begin{abstract}
Recently, epigenetic-mediated mechanisms - which involve heritable changes in gene expression in the absence of alterations in DNA sequences - have been proposed as contributing to asthma. In this issue of the JCI, Hollingsworth and colleagues report on the effect of prenatal maternal dietary intake of methyl donors on the risk of allergic airway disease in offspring in mice and show that these effects involve epigenetic regulation (see the related article beginning on page 3462). Supplementation of the maternal diet with methyl donors was associated with greater airway allergic inflammation and IgE production in F1 and, to some extent, F2 progeny. Site-specific differences in DNA methylation and reduced transcriptional activity were detected. If these findings are confirmed, a new paradigm for asthma pathogenesis may be emerging.
\end{abstract}

Conflict of interest: The author has declared that no conflict of interest exists.

Citation for this article: J. Clin. Invest. 118:3265-3268 (2008). doi:10.1172/JCI37171.
More and more, it seems that our traditional view of asthma as a complex disease that is mediated by a genetic predisposition and childhood or later environmental exposures needs updating. At this point, a mounting body of literature has established that prenatal exposures can influence the risk for developing asthma (1). This link has been most firmly documented in epidemiological studies of prenatal exposure to cigarette smoke and subsequent wheeze. For example, in a large prospective Danish cohort study of over 11,000 children, maternal smoking at the 36th week of gestation was associated with transient wheezing in children before age 3 years (2). In a Stockholm cohort of over 4,000 newborns, maternal smoking during but not following pregnancy was associated with an increased risk of recurrent wheezing in offspring up to age two years (3). In mouse 